\title{
LA EDU-COMUNICACIÓN: UNA NECESIDAD SENTIDA, UNA APUESTA URGENTE
}

\author{
A EDU-COMUNICAÇÃO: UMA NECESSIDADE \\ SENTIDA, UMA APOSTA URGENTE \\ THE EDU-COMMUNICATION: \\ A FELT NEED, AN URGENT STAKE \\ L'EDU-COMMUNICATION : UN BESOIN \\ RESSENTI, UN ENJEU URGENT
}

José Ignacio Aguaded Gómez *

\begin{abstract}
RESUMEN
Vivimos, sin duda, en la sociedad de la información; los medios de comunicación se han convertido en nuestro referente más cercano y la virtualidad compite con la propia realidad en la construcción de las identidades. Por ello, la educación en medios de comunicación se convierte en una pieza clave de una sociedad democrática que busque una ciudadanía responsable e inteligente. En este trabajo apuntamos algunas notas para justificarla en el contexto actual, esbozar su conceptualización, sus objetivos y contenidos, a fin de darle entidad como un ámbito de estudio que supere la visión excesivamente tecnológica e instrumental que hoy tiene, fruto de las modas e idolatrización de los avances tecnológicos.
\end{abstract}

Palabras-clave: Sociedad de la información. Edu-comunicación. Sociedad democrática. Ciudadanía inteligente.

* Grupo Comunicar / Universidad de Huelva - España. Vicerrector de Tecnologías, Innovación y Calidad de la Universidad de Huelva. Profesor de Educación y Comunicación. Doctor en Psicopedagogía (aguaded@uhu.es; director@grupocomunicar.com). 
El fenómeno comunicativo es, sin duda, la nota más trascendental y significativa que caracteriza la sociedad del mundo contemporáneo. Aunque en todos los períodos de la historia el hombre se ha servido de instrumentos para comunicarse, la magnificación y universalización de los medios y recursos del mundo contemporáneo han hecho que esta actividad tan específica del género humano se haga especialmente singular en nuestros días. Sin embargo, el hecho comunicativo contemporáneo no se puede entender en su complejidad se no lo situamos plenamente dentro de la revolución científica y tecnológica a la que estamos asistiendo: innovaciones técnicas cada vez más sorprendentes, mejoras tecnológicas en las condiciones laborales, aparatos de todo tipo en nuestros hogares, un maquinismo creciente en todos los órdenes de la vida, el aumento de las horas dedicadas al ocio... Esta superación, al menos parcial, de las barreras del tiempo, del espacio e incluso de la materia, gracias al efecto de los medios de comunicación y las tecnologías de la comunicación y la información, están configurando, sin duda, un nuevo modelo de ser humano y de sociedad, como nunca había ocurrido antes en la historia y todo ello a un ritmo de vértigo.

Responder reflexivamente a esta nueva sociedad de la información es cuando menos, una exigencia que todos los ciudadanos y ciudadanas deberían plantearse. Nadie duda ya ni cuestiona la poderosa influencia cuantitativa y cualitativa que sobre los ciudadanos ejercen los medios de comunicación. Aunque si bien es cierto, los estudios de audiencia han puesto en evidencia que ésta no es plana ni uniforme y ni siquiera directa, dependiendo de muchos condicionantes personales, sociales y contextuales, la verdad es que los medios son en parte la «atmósfera que respiramos».

La presencia de los medios a nivel social es especialmente sensible en las nuevas generaciones. De estas complejas relaciones de los niños y jóvenes con los medios de comunicación surge la necesidad de planificar y proyectar una educación para el conocimiento de estos nuevos lenguajes en el contexto de una sociedad cada vez más mediática, e indagar y reflexionar sobre cómo la educación, en general, y la enseñanza en particular, han de responder al papel central que los medios de comunicación juegan en la vida de los jóvenes.

En este sentido, creemos esencial explicitar y justificar las razones para la integración curricular de los medios de comunicación, definiendo qué entendemos por «educación en medios de comunicación», estableciendo no sólo su conceptualización, sino analizando sus objetivos, sus contenidos, y los marcos conceptuales en los que se apoya... de manera que la educación en medios de comunicación se entienda como un ámbito de estudio para la educación de la "competencia comunicativa», que supere la visión excesivamente tecnológica e instrumental, que fruto de las modas y lo atractivo de los avances tecnológicos, a menudo confunde y distorsiona las inherentes características y cualidades que los medios tienen de cara a la educación. 


\section{RAZONES PARA UNA EDUCACIÓN EN MEDIOS DE COMUNICACIÓN}

Muchos investigadores señalan la contradicción y paradoja que se produce entre la relevancia social de la información y su escasa presencia en las aulas, pues «mientras los sistemas de comunicación y el flujo de la información son elementos cada vez más vitales para la actividad social, económica y política en todos los niveles, la educación audiovisual sigue siendo algo marginal en los sistemas educativos de todas las partes» (MASTERMAN, 1993). Es preciso para ello, y siguiendo a este autor, sistematizar algunas ideas fundamentales para definir la necesidad imperiosa de una educación en medios y justificar, por ende, la necesaria integración que estos han de tener en el ámbito educativo:

a) Los niveles de consumo (omnipresencia). Las altas tasas de dedicación a los medios (la radio, el cine, Internet, la prensa y especialmente la televisión) y la creciente saturación de éstos en la sociedad contemporánea es quizás la primera y principal causa que justifica que éstos no estén ausentes, o mejor totalmente ignorados, en los centros educativos. La cantidad de horas de consumo de cualquier niño o joven de hoy en cine, radio, televisión, revistas, ordenadores, publicidad, vídeos... es realmente abrumadora. Ahora bien, lo ciertamente preocupante no es sólo la cantidad de tiempo de ocio dedicada a los medios, sino, cómo nuestra experiencia personal depende de los medios y está saturada de los mismos.

b) Los contenidos mediáticos. Jacques Gonnet (1995, p. 35) reivindica el papel del uso de los medios para el conocimiento de la actualidad y la formación democrática. Sin embargo, la realidad mediática a la que asistimos en todo el mundo es el aumento progresivo de la manipulación y la fabricación de la información y su propagación por los medios, así como él éxito de los programas de peor calidad cultural y social: los programas llamados «basura». Pero además, los medios generan claramente una desigualdad considerable entre los que ostentan el poder de la información y los que la consumen de manera indiscriminada e inocentemente. En una sociedad cada vez más mediatizada, la influencia de la comunicación y la información es lógicamente más decisiva a la hora de defendernos de los graves excesos de la manipulación en los medios con fines políticos. El vertiginoso incremento de las presiones nacionales e internacionales para privatizar la comunicación. La información se entiende como un bien de consumo y ello conlleva al desarrollo de empresas corporativas mundiales que controlan los sistemas, tecnologías y empresas de la comunicación.

c) Los niveles de penetración (omnipotencia). La importancia ideológica de los medios y su influencia como empresas de concienciación. Además de nuestro elevado consumo y atención a los medios, ellos moldean nuestras percepciones e ideas, construyéndonos una realidad ficticia, como verdaderas «empresas de concienciación», con lo que debemos superar «la creencia común de que la función primera de los medios es proporcionar noticias, información y entretenimiento a sus audiencias» $y$, en consecuencia, afrontar una lectura activa de sus mensajes. 
Surge así la necesidad de educar a los alumnos para que hagan frente a las exigencias del futuro. Las instituciones escolares han ignorado con excesiva frecuencia la educación de los hechos presentes, los cambios culturales, políticos y de todo orden del mundo contemporáneo. Por tanto, el debate no ha de estar sólo en la incorporación de los medios en la escuela, sino «el tipo de formación cultural que queremos potenciar..., cómo integrar esa cultura mediática en las escuelas, cómo transformamos a los medios de comunicación en objeto de estudio y análisis curricular y cómo logramos que los estudiantes transfieran este conocimiento a su vida cotidiana", en definitiva, en plantearnos una filosofía y modelo de escuela (AGUADED, 1993).

Si partimos de la base de que los planteamientos de los docentes, en este campo de la educación audiovisual, no coinciden, en la mayoría de los casos, con las actuaciones y pensamientos de la sociedad en general y de las nuevas generaciones en particular, la justificación de la educación para la comunicación se hace básica y por ello la finalidad de la educación en medios de comunicación no ha de ser otra de que «los alumnos comprendan de manera activa y consciente, en lugar de pasiva e inconscientemente, el lugar en que se encuentran y que capten el sentido de los textos de los medios de comunicación social» (SHEPHERD, 1993).

\section{LA INSERCIÓN CURRICULAR DE LA EDUCACIÓN EN MEDIOS}

La inserción curricular de la educación en medios de comunicación presenta diferentes alternativas como reflejo de las distintas corrientes y tradiciones. En síntesis, se defiende por un lado la opción de una asignatura independiente y en igualdad de condiciones con el resto de las materias curriculares, y en otra línea el tratamiento transversal, justificando la necesidad de la interdisciplinariedad, en que los medios exceden las áreas disciplinares tradicionales.

La integración de la educación en medios de comunicación dentro del currículum escolar, en suma, se puede establecer desde tres enfoques diferenciados, como apunta Area (1995), sintetizando aportes anteriores:

- Área o asignatura independiente equiparable al resto de las materias del currículum, con espacios propios para su estudio, a menudo como una asignatura de segundo orden y en conflicto con otras asignaturas por sus metodologías más innovadoras.

- Contenido (conceptual, procedimental o actitudinal) dentro de los bloques de las áreas curriculares concretas. De esta forma se rompe el planteamiento global de la comunicación y en la mayoría de los casos los medios se reducen a su vertiente de auxiliares y recursos didácticos, con lo que la función que consideramos más genuina, de análisis de la comunicación contemporánea desde una vertiente activa, esto es, crítica y lúdica, se pierde. Además, esta integración parcial depende siempre de la específica preocupación del profesorado por esta área de conocimiento. 
- Tema transversal a lo largo de todo el currículum. La administración educativa española, en el proceso de la reformas educativas derivada han apostado decididamente por la inclusión de la «educación en medios de comunicación» en los diseños curriculares de los Ministerios de Educación. Tanto los objetivos de etapas y ciclos, como los de las distintas áreas recogen implícita y explícitamente - aunque de una manera muy desigual - la necesidad del uso de los medios de comunicación como nuevos contenidos relevantes, como lenguajes de comprensión e interpretación de la realidad y como nuevos medios al alcance de la mano. Sin embargo, en la mayoría de los países, la «educación en medios de comunicación» no aparece formalmente como un área transversal, junto a los otros ámbitos reconocidos como tales, esto es, la educación ambiental, la educación del consumidor, la educación para la paz, etc.

Una enseñanza específica sobre los medios no tendría un impacto efectivo si sus métodos no se introducen en todas las otras disciplinas y no influyen en el análisis de los productos mediáticos. Sin embargo, coincidimos con Masterman (1993) en que el estudio de los medios como disciplina específica "presenta enormes ventajas", ya que hay razones imperiosas que han llevado a los especialistas a pensar que la integración curricular de disciplinas específicas sobre la educación en los medios es la mejor manera de afianzar la educación para la comunicación, en los niveles de enseñanza superiores, mientras que en educación primaria la opción más válida parece ser su integración interdisciplinar.

La educación en medios de comunicación participa ampliamente de lo que ha venido en llamarse "temas transversales», ya que los alumnos comprenden los problemas cruciales de la sociedad, elaborando un juicio crítico sobre ellos a partir del trabajo con los medios, con lo cual se determina el para qué de la educación. Además, la integración de los medios en el currículum fomenta no sólo capacidades intelectuales, sino también relaciones interpersonales, valores, actitudes, normas, estrategias y procedimientos, así como la inserción en el mundo social. Por otro lado, la educación en medios parte de la toma de conciencia de una problemática social surgida en las últimas generaciones: el progreso y el avance tecnológico, y por tanto, refleja los intereses, los conocimientos y las ideas previas del alumnado.

Es fundamental para definir las señas de identidad del centro educativo, dado que un uso integral de este eje transversal supone la implicación de todos los sectores de la comunidad escolar y su inclusión en el proyecto de centro. Además, se favorece la relación de la escuela con su entorno, ya que los medios de comunicación vinculan a la escuela y la familia en un proceso compartido de aprendizajes y apropiaciones. En suma, la educación en medios abarca a todos los niveles y áreas del sistema educativo, trascendiendo el marco curricular.

El tratamiento de los medios de comunicación, para el acercamiento de los alumnos a la realidad cotidiana, no se limita sólo a contenidos informativos o conceptuales, sino 
que debe estimular y atender, puesto que sus características lo facilitan, con una dinámica didáctica adecuada y centrada en el alumno, la motivación, los valores, la interculturalidad, el pensamiento crítico, la actitud investigadora, la interdisciplinariedad y el descubrimiento y la creación de sus propios mensajes de respuesta al medio.

Conocer críticamente los medios y saber emplear los nuevos lenguajes de forma creativa rompe definitivamente el distanciamiento entre lo que la escuela enseña y lo que los niños y chicos viven en sus hogares y en la calle (AGUADED; CONTÍN, 2002). El empleo de los medios en todos los ámbitos curriculares, tanto horizontal como verticalmente, permite afirmar (CONTRAPUNT, 1994) que la «educación en comunicación» más que un eje transversal, sea una "transversal de transversales», por «ser un vehículo poderoso de integración de los saberes de las distintas áreas transversales».

La inclusión o no de la «educación en medios de comunicación» como eje transversal del currículum refleja un modelo concreto de concepción de la enseñanza, así como un tipo definido de conceptualización del uso didáctico - global o parcial - de los medios de comunicación en el aula.

\section{EDUCACIÓN EN MEDIOS DE COMUNICACIÓN}

El fenómeno del «analfabetismo audiovisual», tal como apunta Tyner (1993) es una realidad palpable en nuestra sociedad. Cada vez más investigaciones y estudios demuestran que el consumo masivo e indiscriminado de los medios no lleva parejo, más bien al contrario, un conocimiento de los códigos del lenguaje audiovisual, provocando situaciones de indefensión ante sus mensajes (AGUADED, 1999; PÉREZ TORNERO, 1994).

La educación en medios de comunicación no puede reducirse al uso de los medios en cuanto tecnologías al servicio del proceso de enseñanza-aprendizaje, por muy interesantes y didácticas que éstas puedan ser, o por lo mucho que motiven y faciliten este proceso, ni tampoco es posible entenderla como una formación específica para el ejercicio de determinadas profesiones en el campo de las comunicaciones sociales. Su sentido y finalidad ha de ser la formación de la conciencia crítica y el desarrollo de actitudes activas y creativas en los alumnos para conocer y comprender los envolventes procesos de comunicación que vive la sociedad de hoy.

Concretamente, la educación en medios de comunicación, en el marco de una enseñanza de calidad, tendrá que definirse atendiendo a las expectativas que los niños y jóvenes tienen en cuanto a los medios. No se trata tanto de defenderlos de ellos, ya que aunque es cierto el uso y abuso indiscriminado que se hace de los mismos, no se deben obviar las enormes ventajas de su expresión y comunicación. También es necesario considerar el análisis del contenido y la relación que mantiene el sujeto receptor con los medios de comunicación, de manera que se pueda establecer una confrontación entre la propuesta cultural de los medios - como parte de la sociedad -, y las percepciones 
espontáneas que se puedan tener, para esclarecer con ello las convergencias y divergencias. Por otro lado, hay que tener presente una formación de personas que sean conocedoras de los nuevos lenguajes audiovisuales de nuestra sociedad y sean capaces de apropiarse críticamente de ellos y de emplearlos creativa y activamente como canales personales de comunicación. En esta línea, hay que tender hacia una acción pedagógico-didáctica, adecuadamente organizada que promueva una actitud crítica, una comprensión del lenguaje audiovisual, de la estructura y función que los medios representan y además incentive las capacidades y actitudes de los alumnos. En suma, la educación en medios debe tender hacia una concepción globalizada del fenómeno educativo en su doble interacción con los medios, como objeto de estudio dentro de la escuela y como instrumento al servicio de un modelo alternativo de comunicación educativa.

La Unesco, en 1979, establecía una definición que se ha considerado como el patrón de referencia para este ámbito de conocimiento: «Todas las formas de estudiar, aprender y enseñar a todos los niveles (...) y en toda circunstancia, la historia, la creación, la utilización y la educación de los medios de comunicación como artes prácticas y técnicas, así como el lugar que ocupan los medios de comunicación en la sociedad, su repercusión social, las consecuencias de la comunicación mediatizada, la participación, la modificación que producen en el modo de percibir, el papel del trabajo creador y el acceso a los medios de comunicación» (UNESCO, 1984). La educación en medios de comunicación puede definirse, por tanto, como «la educación de los niños y niñas y jóvenes de ambos sexos para que sean receptores críticos, selectivos y activos ante los mensajes que reciben a través de los distintos medios de comunicación» (BERNABEU, 1994), de manera que en el centro se desarrolle una verdadera «cultura comunicativa».

Los destinatarios de este nuevo «eje transversal» de la enseñanza pueden ser los maestros y profesores de todos los niveles del sistema educativo y de todas las especialidades curriculares; os niños y jóvenes, expuestos diariamente a una «dosis» audiovisual considerable, sin conocimiento ni análisis de estos nuevos códigos de interpretación y expresión del mundo. Pero también los grupos y responsables comunitarios (educadores, animadores, asociaciones, etc.), preferentemente de ámbitos populares.

Por último, indicar que si bien hay un gran consenso mundial, tanto en la necesidad de poner en marcha programas de educación para la comunicación, como en los objetivos y metodologías, hay en cambio una gran variedad de denominaciones para titular esta importante parcela curricular. El término «educación para los medios» se emplea actualmente en Iberoamérica. Como objeción al mismo, cabe señalar que, en un estricto sentido, la palabra «medios» en castellano, como señala Sancho (1995), es excesivamente amplia en el ámbito general e incluso a escala docente, recogiendo otros recursos didácticos ajenos a la comunicación social. En los últimos años se ha utilizado con frecuencia el término "educación en materia de comunicación», recogiendo explícitamente el título de la traducción al castellano del libro de la Unesco (1984): «éducation aux médias», 
traducido por la propia Organización en el mismo año. Aunque la denominación está ya asentada, tiene como principal handicap la amplitud del concepto de "comunicación" que al igual que «medios» en solitario hace referencia a otras muchas realidades, además de la comunicación social y sus medios de transmisión. Como variante se encuentra el de «educación para la comunicación», que mantiene la misma amplitud semántica, pero dando en sí cabida la educación en los medios de comunicación. Hay otras denominaciones que en las últimas décadas han ido imponiéndose con mayor o menor asentamiento, vinculadas a la especificidad de ciertas corrientes.

El término que nos parece más genérico, «educación en medios de comunicación» o bien en plan sintético, «edu-comunicación»-, recoge, mejor que ningún otro, la traducción de la acepción que tiene en otros países la palabra inglesa «media». Además, consideramos que la preposición «en» es la que mejor define el sentido de la inclusión de los medios de comunicación con una finalidad crítica en la enseñanza, frente al sentido finalista - y consideramos también reducionista - de la preposición "para».

\section{METASY FINALIDADES DE LA EDU-COMUNICACIÓN}

Si partimos de la base de que cualquier proceso de enseñanza-aprendizaje requiere una planificación, es evidente la necesidad de justificar cuáles son los objetivos de la integración curricular de los medios, admitiendo que las aspiraciones de la edu-comunicación no pueden ser esencialmente conceptuales, sino más bien actitudinales y valorativas.

El aspecto clave que justifica la educación en medios de comunicación es el desarrollo de la capacidad de análisis y crítica del alumno y para ello habrá que favorecer una toma de conciencia ante los medios, que facilite esa necesaria distancia para apreciar los mensajes de estos. En consecuencia, la tarea verdaderamente importante y difícil del profesor de medios será fomentar en sus alumnos la confianza en sí mismos y la madurez crítica suficientes para que puedan aplicar sus juicios de valor. Por tanto los objetivos irán encaminados a: descubrir los mecanismos, simbolismos y estrategias que emplean los medios para construir la realidad y seducirnos, así como a seleccionar y descubrir las intenciones ocultas y valores que los medios, con una aureola de transparencia, transmiten.

Además, será muy importante identificar y comprender las principales funciones y propósitos de los medios, especialmente, la información, la formación y el entretenimiento, como conocer el funcionamiento de los medios, el modo en que se constituyen como industrias culturales, los intereses y poderes asociados y su papel en la sociedad. La educación en medios ha de procurar, por otro lado, desarrollar la creatividad, la innovación y la cooperación, utilizando la expresión y comprensión de los medios como nuevo lenguaje comunicativo, utilizando para ello técnicas de investigación y documentación, mediante la búsqueda, selección, e interpretación de la información. 
En suma, se busca educar para el pluralismo y la tolerancia, a través del contacto con la diversidad de culturas, de expresiones y de formas de vida, y todo ello introduciendo una metodología participativa en el aula con múltiples recursos y cauces de información y comunicación entre los distintos miembros de la comunidad escolar y líneas de cooperación e intercambio con el exterior.

La educación en medios ha de crear espacios educativos que faciliten el aprendizaje, mediante el acceso a la información y a la producción de comunicación por parte de profesores y alumnos, valorando la función social de los medios de comunicación de masas y de los nuevos medios técnicos y su importancia como instrumentos de cooperación y solidaridad.

En definitiva, la educación en medios deberá plantearse como una educación «multidimensional» que sitúe al alumno ante el proceso de la comunicación como emisor activo y como receptor crítico, fomente la creatividad y sensibilidad hacia los nuevos lenguajes, enseñe a decodificar sus códigos y a analizar críticamente sus contenidos, enriquezca los criterios de juicio, aumentando su autonomía y distanciamiento ante los mensajes de los medios, abriendo nuevas perspectivas hacia otras culturas, favorezca la expresión y comunicación mediante el lenguaje audiovisual, con la creación y elaboración de productos mediáticos, y desarrolle la socialización y la participación, en una actitud de apertura, de plena libertad de expresión y de rechazo de todo adoctrinamiento desde los medios.

\section{LOS CONTENIDOS}

El proceso de enseñanza-aprendizaje que determina la edu-comunicación requiere una selección de contenidos acorde con un modelo de aprendizaje en el que la recepción, la reflexión y la acción/respuesta sean los ejes fundamentales, de tal manera que las tres grandes dimensiones en las que se han de delimitar los contenidos de los medios sean: la recepción, su análisis y el desarrollo de destrezas comunicativas y expresivas.

Los medios construyen la realidad, son símbolos, signos, sistemas, que hay que interpretar, ya que no son más que un reflejo, una representación. Por lo tanto los principios genéricos y los aspectos claves que, con mayor o menor insistencia, se han de tener presentes se establecen en torno a la necesidad de fomentar el conocimiento crítico y creativo, esto es, activo con los medios de comunicación. Así se deduce que las áreas esenciales que ha de contener la educación para los medios se han de centrar en:

- El texto que significan los medios; es decir, analizar las producciones mediáticas en sus diferentes tipos, géneros, códigos y estructuras, así como en sus significados denotativos y connotativos, estableciendo ideologías y valores y sus consiguientes interacciones.

- Quién o quienes lo reciben. El significado de los textos no está tanto en factores inherentes al texto en sí como en el significado que otorgan los receptores. Cuestiones como 
múltiples significados del texto, los valores y sesgos implícitos en esos significados, las elecciones y selecciones, el uso, el disfrute, la cultura, el género, etc. han ido adquiriendo más importancia en los últimos años, como consecuencia de investigaciones recientes, con lo que parece fundamental dotar al alumno de habilidades que le permitan una lectura activa sobre los medios.

- La propia producción de los textos mediáticos. La educación en medios ha de facilitar el conocimiento de "cómo se ha producido un texto, quiénes lo han producido, por qué, en qué circunstancias y en qué contexto se ha producido» (SHEPHERD, 1993). Por ello en este área hay que profundizar en contenidos como las tecnologías, las prácticas de producción utilizadas, aspectos económicos de propiedad y control, su distribución, etc.

La educación en medios debe facilitar el conocimiento, las estrategias, destrezas y actitudes necesarios para la lectura de estos textos de forma personal y crítica, a la vez que las respuestas activas a los mismos. Nos interesa conocer "cómo funcionan los medios, cómo producen significado, cómo son parte de la industria de construcción de la realidad y cómo esa supuesta realidad ofertada por los medios es interpretada por quienes la reciben" (MASTERMAN, citado por AREA, 1995). En definitiva, los alumnos deben aprender con una metodología de investigación y construcción del conocimiento que acompase ejercicios de lectura crítica de los medios, con actividades de producción y expresión personal.

Considerar los medios como simples instrumentos tecnológicos facilitadores de la enseñanza, nos aleja de los modelos conceptuales que establecen como elementos claves de la educación en medios de comunicación el estudio de la semiótica, la ideología, los contextos sociales de la producción y el consumo y el público destinatario, además del planteamiento de transversalidad. Ello supone una concepción reducionista de la educación en medios de comunicación que ha traído consigo la separación artificial del uso de los medios (prensa, por un lado; y las llamadas «nuevas tecnologías», por otro) sin permitir entrar en profundidad en una visión global de la comunicación y en las aportaciones que este eje transversal, como ámbito de conocimiento específico.

Sin embargo parece claro, a la luz de las más recientes corrientes e investigaciones, que la edu-comunicación comprende unos nuevos lenguajes, con nuevos códigos de lectura, de comprensión e interpretación, que van más allá de la suma del lenguaje escrito con el visual, y que suponen por ello una auténtica revolución comunicativa, al aportar una nueva codificación de la realidad, más allá de los propios medios. En consecuencia, engloba necesariamente también todo el conjunto de tecnologías de la información y la comunicación que progresivamente van sumándose a la vida diaria, gracias al impresionante avance de las ciencias. Son estas tecnologías-punta las que sin duda han ido configurando este nuevo marco de conocimiento y estos nuevos lenguajes. Ahora bien, teniendo presente que los instrumentos materiales no son más que la base y los soportes, que 
responden a una realidad mucho más compleja, pues su uso no tiene sentido si no es encua-drado dentro de un contexto más amplio, como es el de la comunicación del mundo moderno, con sus nuevos lenguajes y medios y su novedosa conceptualización de la realidad.

Por todo ello, una óptima propuesta de la educación en medios de comunicación debe centrarse, como indica Bernabeu (1994), en dos ejes centrales: la formación de receptores críticos y el fomento de la cultura comunicativa. Al mismo tiempo, hemos de diferenciar, siguiendo a esta autora:

- «La educación en los medios» o conceptos. El estudio de los medios en sí mismos y en este sentido, hay que entender la lectura crítica que permite valorar e interpretar los mensajes, seleccionándolos como receptor activo.

- «La educación con los medios» o procedimientos: estrategias que permitan el desarrollo de técnicas de trabajo intelectual e investigación, el conocimiento del alfabeto audiovisual, los elementos que lo componen, sus reglas, etc.

- «La educación ante los medios» o actitudes: consumo selectivo, espíritu crítico y criterio personal. Se tarta de formar en torno a la influencia de los medios de comunicación en la sociedad y en la propia vida.

\section{A MANERA DE CONCLUSIÓN}

La educación en la sociedad audiovisual debe contemplar la específica situación que hemos tratado de exponer, y, partiendo de su análisis, concretarse en un proyecto educativo concreto, con una filosofía educativa, que adecue las prácticas y actuaciones escolares a las nuevas demandas sociales (CREEL; OROZCO, 1993).

En virtud de esas coordenadas sociales y educativas, los medios adquieren roles específicos en sus relaciones con los procesos de enseñanza-aprendizaje. Así, los medios, en un contexto de pedagogía transmisiva, como la que hasta ahora ha impregnado prácticamente el quehacer educativo en casi todas las regiones del mundo, pueden no ser más que meros auxiliares que complementan la función informativa de los profesores, ampliando sin el campo de conocimientos conceptuales a través del apoyo del lenguaje audiovisual. Éste es un primer paso, sin duda, pero muy limitado, porque donde realmente cobra sentido la dimensión comunicativa es cuando se convierte en el eje de una nueva conceptualización de la educación en la sociedad audiovisual en que nos ha tocado vivir. "La educación emancipadora tiene una epistemología alternativa que, en contraposición al conocimiento objetivo, se basa en el conocimiento comunicado. Este conocimiento es generador y no consumidor; se preocupa de la percepción y no de la recepción... En un sistema así la inteligencia constituye un proceso y no un producto» (CRÍTICOS, 1993, citando a STANTON). 
De esta manera la sociedad audiovisual demanda, frente a los estilos tecnicistas y pragmáticos que han imperado como modelos educativos, el «aprendizaje experiencial reflexivo", que supere la educación bancaria (FREIRE) de depositar conocimientos dentro de las cabezas de los alumnos y que promueva alumnos más críticos y creativos con su entorno, conscientes de su realidad y capaces de actuar libre, autónoma y juiciosamente. Pensamos, que el papel de los medios de comunicación en esta enseñanza crítica y de valores tiene que ser necesariamente crucial.

En este sentido, se pueden citar como estrategias más válidas «el pensamiento crítico, los modelos indagadores, los enfoques de estudios culturales, la educación de los valores, las estrategias interdisciplinarias, las experiencias creativas y la pedagogía democrática y centrada en el alumno» (TYNER, 1993).

Una sociedad cada vez más consumidora de medios - que de forma paradójica, apenas si se preocupa por fomentar aptitudes para el conocimiento racional de los códigos y lenguajes de éstos -, ha de poner en funcionamiento propuestas didácticas que permitan una intervención consciente del sistema educativo para "alfabetizar» a los chicos y chicas de hoy como consumidores y usuarios de los nuevos lenguajes de la comunicación y la información, con plena conciencia de uso y con potencialidad para su utilización crítica y creativa.

La importancia social y personal de la comunicación audiovisual en el marco de la vida moderna y la necesidad de desarrollar propuestas críticas y creativas en el ámbito educativo para saber comprender, interpretar y utilizar los medios, requiere la presencia de éstos en los diferentes ámbitos del saber, puesto que no se trata sólo de adquirir conocimientos, ni de promover actitudes, sino de fomentar técnicas y procedimientos que permitan al alumnado su análisis y uso como lenguajes propios.

Los medios de comunicación no sólo presentan adecuadas estrategias para favorecer los objetivos del sistema educativo, sino que al mismo tiempo ofrecen propuestas metodológicas, al hilo de las corrientes didácticas en boga. En este sentido, el aprendizaje significativo, la globalización de los contenidos, la actividad del discente, la fundamentación en el entorno, la diversidad de los recursos, el inicio de los procesos a partir de las ideas previas, el carácter formativo de los procedimientos de valoración y evaluación, el rol de orientador y estimulador de los docentes, la flexibilidad organizativa, la adaptación curricular a las necesidades particulares del alumnado, la interdisciplinariedad curricular, la importancia de valores y actitudes... encuentran en los medios de comunicación unos recursos variados y de fuerte impacto y valor didáctico.

Los medios, en este contexto didáctico, acercan el proceso de aprendizaje al modelo investigador. Frente al estilo transmisivo y centrado en los contenidos, que hemos visto que no responde a las exigencias de la sociedad audiovisual, los medios favorecen la investigación y exploración de la realidad por parte de los alumnos y alumnas, que pueden aprender en un proceso de descubrimiento, al tiempo que son protagonistas de 
su aprendizaje. Los medios tanto como auxiliares didácticos, como técnicas de trabajo o ámbitos de conocimiento diversifican las fuentes de información, partiendo de la «actualidad» y del entorno, ofreciendo informaciones globales que afectan integralmente a todas las áreas curriculares. Definitivamente, permiten cambiar la dinámica tradicional del aula, reduciendo la función informativa del profesor y reservándole competencias didácticas más genuinas, como la de planificación, motivación y evaluación. Coincidimos, finalmente, con Len Masterman (1993) en que exigen nuevas formas de trabajar, en la línea de una enseñanza no jerárquica, «que fomente la reflexión y el pensamiento crítico y que, al mismo tiempo, sea lo más viva, democrática, centrada en el grupo y orientada a la acción que el profesor pueda conseguir».

La sociedad audiovisual reclama un contexto educativo en el que los medios han de jugar un papel crucial. McLuhan afirmaba que la pedagogía actual no se corresponde con la era de la electricidad, sino que se quedó en la era de la escritura. Vallet, por otra parte, proclamaba nuestro analfabetismo de cara a los medios de comunicación. Si nuestra escuela ha de preparar a los alumnos de hoy a vivir en la sociedad de forma autónoma y libre, no puede seguir ignorando los medios. No queda otra alternativa que integrarlos didácticamente y sacar de ellos todo lo positivo que puedan ofrecernos La pedagogía audiovisual tiene que entrar en la institución escolar, si se quiere potenciar las dimensiones lúdicas, críticas y creativas de las nuevas generaciones para su adecuada inserción en la sociedad audiovisual. Se trata de que la educación establezca sistemas de comunicación con su entorno y procese la información del contexto de un modo útil a sus fines y proyecte sus mensajes hacia fuera.

\section{Referencias}

AGUADED, José Ignacio. Sociedad consumista y medios de comunicación, Comunicar, n. 7; p. 8-9, 1996.

1999.

. Convivir con la televisión. Familia, educación y recepción te-levisiva. Barcelona: Piados,

; CABERO, Julio (Eds.). Educar en red. Málaga: Aljibe, 2002.

; CONTÍN, Silvia (Coords.). Jóvenes, aulas y medios de comunicación. Buenos Aires: La Crujía, 2002.

AREA, Manuel. La educación de los medios de comunicación y su integración en el currículum, Píxel-Bit, n. 4, p. 5-19, 1995.

BERNABEU, Natalia. Tratamiento transversal de la educación en materia de comunicación. In: UNED: Medios de comunicación y educación. Córdoba (España): Centro Asociado, 1994. p. 129144 .

CONTRAPUNT, Equipo. Los contenidos de la educación en materia de Comunicación. Comunicar, n. 3, p. 167, 1994. 
CREEL, Mercedes; OROZCO, Guillermo. El proceso de la recepción y la educación para los medios: una estrategia de investigación con público femenino. In: APARICI, Roberto (Coord.). La revolución de los medios audiovisuales. Madrid: De La Torre, 1993. p. 199-220.

CRÍTICOS, Costa. Aprendizaje experiencial y transformación social para una enseñanza futura sin apartheid. In: APARICI, Roberto. (Coord.). La revolución de los medios audiovisuales. Madrid: De La Torre, 1993. p. 79-92.

GONNET, Jacques. De l'actualité à l'école. Pour des ateliers de démocratie. París: Armand Colin, 1995.

GUTIÉRREZ, Alfonso. Educación multimedia y nuevas tecnologías. Madrid: De la Torre, 1997.

IRIARTE, P. Gregorio; ORSINI PUENTE, Martha. Educación y medios de comunicación. Cochabamba (Bolivia): Cepromi, 1996.

MASTERMAN, Len. La enseñanza de los medios de comunicación. Madrid: De La Torre, 1993 (Traducción española de Teaching the media).

PÉREZ TORNERO, José Manuel. El desafío educativo de la televisión. Barcelona: Piados, 1994.

SANCHO, Juana. ¿El medio es el mensaje o el mensaje es el medio? El caso de las tecnologías de la información y la comunicación. Pixel-Bit, n. 5, p. 51-67, 1995.

SHEPHERD, Rick. Raíces: origen y desarrollo de los profesores de medios. In: APARICI, Roberto (Coord.). La revolución de los medios audiovisuales. Madrid: De La Torre, 1993. p. 135151.

THESING, Josef; PRIESS, Frank. Globalización, democracia y medios de comunicación. Buenos Aires: Ciedla, Konrad-Adenauer, 1999.

TYNER, Katheen. Alfabetización audiovisual. El desafío de fin de siglo. In: APARICI, Roberto (Coord.). La revolución de los medios audiovisuales. Madrid: De La Torre, 1993. p 171-197.

UNESCO. La educación en materia de comunicación. París: UNESCO, 1984. 


\section{A Edu-comunicação: uma necessidade sentida, uma aposta urgente}

Resumo

Vivemos, sem dúvida, na sociedade da informação; os meios de comunicação se transformaram em nosso referente mais próximo e a virtualidade compete com a própria realidade na construção das identidades. Por isso, a educação em meios de comunicação se transforma em uma peça chave de uma sociedade democrática que busca uma cidadania responsável e inteligente. Neste trabalho apontamos algumas notas para justificá-la no contexto atual, esboçar sua conceitualização, seus objetivos e conteúdos, a fim de dar-lhe entidade como um campo de estudo que supere a visão excessivamente tecnológica e instrumental que se tem hoje, fruto das modas e idolatrização dos avanços tecnológicos.

Palavras-chave: Sociedade da informação. Edu-comunicação. Sociedade democrática. Cidadania inteligente.

\section{The Edu-communication: a felt need, an urgent stake}

\section{Abstract}

We most certainly live in an information society where the means of communication have been transformed into our closest reference system and virtuality competes with reality itself in the construction of identities. For this reason, education in means of communication transforms itself into a key instrument of a democratic society which searches for a responsible and intelligent citizenship. In this paper, we point out a few notes to justify it in the present context, draft its conceptualization, its objectives and contents, in order to give it entity as a field of study which overcomes the excessively technological and instrumental vision as occurs nowadays, as a result of the trends and idolatrization of technological progresses.

Keywords: Information society. Edu-communication. Democratic society. Intelligent citizenship.

\section{L'édu-communication : un besoin ressenti, un enjeu urgent}

Résumé

Nous vivons, sans aucun doute, dans la société de l'information où les moyens de communication se sont transformés en notre référence la plus proche et la virtualité fait compétition avec la propre réalité dans la construction des identités. En fonction de cela, l'éducation en moyens de communication se transforme en une pièce clef dans la société démocratique qui cherche une citoyenneté responsable et intelligente. Dans ce travail, nous pointons quelques notes qui la justifie dans le contexte actuel, ébauche sa conceptualisation, ses objectifs et contenus, afin de lui donner une entité comme champs d'étude qui supère la vision excessivement technologique et instrumentale qui existe aujourd'hui, fruit des modes et de l'idolatrie des progrès technologiques.

Mots clefs : Société de l'information. Édu-communication. Société démocratique. Citoyenneté intelligente.

Recebido: 13.03.2007

Aceito: 27.04 .2007 
